\title{
A structural constitutive model considering angular dispersion and waviness of collagen fibres of rabbit facial veins
}

\author{
Aristotelis Agianniotis $^{*}{ }^{\dagger}$, Rana Rezakhaniha ${ }^{\dagger}$, Nikos Stergiopulos
}

\author{
* Correspondence: aristotelis. \\ agianniotis@epfl.ch \\ Institute of Bioengineering, Ecole \\ Polytechnique Fédérale de \\ Lausanne, 1015 Lausanne, \\ Switzerland \\ † Contributed equally
}

\begin{abstract}
Background: Structural constitutive models of vascular wall integrate information on composition and structural arrangements of tissue. In blood vessels, collagen fibres are arranged in coiled and wavy bundles and the individual collagen fibres have a deviation from their mean orientation. A complete structural constitutive model for vascular wall should incorporate both waviness and orientational distribution of fibres. We have previously developed a model, for passive properties of vascular wall, which considers the waviness of collagen fibres. However, to our knowledge there is no structural model of vascular wall which integrates both these features.
\end{abstract}

Methods: In this study, we have suggested a structural strain energy function that incorporates not only the waviness but also the angular dispersion of fibres. We studied the effect of parameters related to the orientational distribution on macromechanical behaviour of tissue during inflation-extension tests. The model was further applied on experimental data from rabbit facial veins.

Results: Our parametric study showed that the model is less sensitive to the orientational dispersion when fibres are mainly oriented circumferentially. The macromechanical response is less sensitive to changes in the mean orientation when fibres are more dispersed. The model accurately fitted the experimental data of veins, while not improving the quality of the fit compared to the model without dispersion. Our results showed that the orientational dispersion of collagen fibres could be compensated by a less abrupt and shifted to higher strain collagen engagement pattern. This should be considered when the model is fitted to experimental data and model parameters are used to study structural modifications of collagen fibre network in physiology and disease.

Conclusions: The presented model incorporates structural features related to waviness and orientational distribution of collagen fibres and thus offers possibilities to better understand the relation between structure and function in the vascular wall. Also, the model can be used to further study mechanically induced collagen remodelling in vascular tissue in health and disease. 


\section{Background}

Constitutive modelling of vascular tissue has been a challenging area for several decades [1,2]. Structural constitutive models, in particular, attempt to integrate information on composition and structural arrangements of tissue to avoid ambiguities in material characterization. In this way, they offer an insight into the function, structure and mechanics of the principal wall components i.e. elastin, collagen and vascular smooth muscle cells. Structural constitutive models have been developed for a variety of tissues and tissue components including blood vessels [3-8], skin [9], pericardium [10], heart valves [11], tendons and ligaments [12], articular cartilage [13].

In blood vessels, collagen fibres appear in coiled and wavy bundles in their unloaded state $[14,15]$ and the individual collagen fibres have a deviation from their mean orientations $[16,17]$. In the media, collagen fibres are strongly co-aligned [17]. Canham et al. [18] reported the angular standard deviation of fibres in the media as $5.2^{\circ}$ in brain arteries and $5.6^{\circ}$ in coronary arteries [18]. However, within the adventitia layer, collagen fibres have large angular dispersion [17]. A complete structural constitutive model for vascular collagen should incorporate both waviness and orientational distribution of fibres.

Perhaps the most complete framework for structural modelling of fibrous tissue has been presented by Lanir et al. [19-21]. In this framework, the total strain energy function (SEF) is assumed to be a result of the collective contribution of the individual fibres linked with tensor transformations from the fibre coordinates to the global tissue coordinates. A number of previous studies have followed this approach and have incorporated waviness $[22,23]$ or orientational distribution of collagen fibres $[10,11,24]$, to study the effects of collagen micro-organization on the macroscopic behaviour of vascular tissue. Other studies have followed a different approach and involved the use of invariants $[22,23,25]$. Yet, to the best of our knowledge, currently, there is no structure-based SEF for the vascular wall, which includes both waviness and angular distribution of collagen fibres and which has been verified using standard inflationextension tests. We have therefore set as goals of this study to, first, extend our previously developed model $[22,23]$ to include both waviness and angular distribution of collagen fibres, second, to perform a parametric study to analyze the effects of orientational distribution parameters on the macro-mechanical behaviour of the vascular tissue and, third, to assess the suitability and importance of including fibres' orientational distribution by applying the model to experimental data from inflation-extension tests.

\section{Methods}

\section{Experimental database}

We have used the experimental set of data from inflation-extension tests, previously reported in our study on rabbit facial veins [26]. The methods were described in detail in the reported manuscript. Briefly, facial veins of rabbits were excised from young animals, the veins were cleaned from the surrounding tissue and the adventitia was removed mechanically. The veins were then mounted on our inflation-extension device and stretched to their in vivo length $(\lambda z=1.62 \pm 0.09)$. After 10 preconditioning cycles, each vein was inflated in the range of $0-15 \mathrm{mmHg}$ and the outer diameter, luminal pressure and longitudinal force were measured. Tests were carried out after adding $80 \mu \mathrm{mol} / \mathrm{L}$ of sodium nitroprusside (SNP) to the bath to relax smooth muscle 
cells. The geometry of the zero load state and zero stress state were measured directly on intact and cut-open vascular rings and the volumetric fractions of each wall component i.e. elastin, collagen and vascular muscle cells were assessed histologically.

\section{Mathematical model}

The mathematical model presented in this work is an extension of our work on venous tissue [26]. We considered the blood vessel as a thick wall circular cylinder which undergoes inflation-extension tests. To develop the model, time dependent effects were ignored and only the pseudo-elastic loading response was considered. Furthermore, the material was assumed to be incompressible and non-linear.

To formulate the strain energy function and in the absence of vascular tone, we considered only passive properties of the vascular wall and therefore separated our constituent-based strain energy function into two parts representing the elastin and collagen components:

$$
\Psi_{\text {passive }}=f_{\text {elast }} \Psi_{\text {elast }}+f_{\text {coll }} \Psi_{\text {coll }}
$$

where $f_{\text {elast }}$ and $f_{\text {coll }}$ are the fractions of wall cross-section area composed of elastin and collagen, and $\Psi_{\text {elast }}$ and $\Psi_{\text {coll }}$ represent the SEF for the network of elastin and collagen fibres, respectively.

\section{Elastin SEF}

We based our SEF of elastin on our previous work on rabbit veins. We modelled elastin as transversely isotropic material, with one family of fibres in the longitudinal direction, embedded in an isotropic matrix with neo-Hookean material properties. We modelled fibres as a one-dimensional material that bears load only along its axis,

$$
\Psi_{\text {elast }}=c_{\text {elast }}^{i}\left(I_{1}-3\right)+c_{\text {elast }}^{a}\left(I_{4}^{\prime \prime}+\frac{2}{\sqrt{I_{4}^{\prime \prime}}}-3\right)
$$

where $c_{\text {elast }}^{i}$ and $c^{a}$ elast represent the modulli for the isotropic and anisotropic elastin components. $I_{1}$ is the first invariant of the Cauchy-Green deformation tensor $C$ and $I_{4}$ " is an invariant of $C$ with respect to $e_{z}$ defined as:

$$
I_{4}^{\prime \prime}=e_{z} \cdot C \cdot e_{z}=\lambda_{z}^{2}
$$

$e_{z}$ being the unit vector in the axial direction.

\section{Collagen SEF}

The constitutive model for collagen used here is based on the work by Lokshin and Lanir on fibrous connective tissue $[9,19,20]$. The framework is therefore founded on the assumption that the gross behaviour of the tissue results from the collective contribution of the individual components.

Schematically, the model for collagen is shown in Figure 1. Collagen fibres are considered wavy in their unloaded state and arranged in two symmetric families of fibres in the circumferential-longitudinal plane. Each fibre is characterized (in the unstrained state) by its directional vector $\boldsymbol{u}$ and its local straightening strain $E^{*}$. The $\boldsymbol{u}$ coincides 


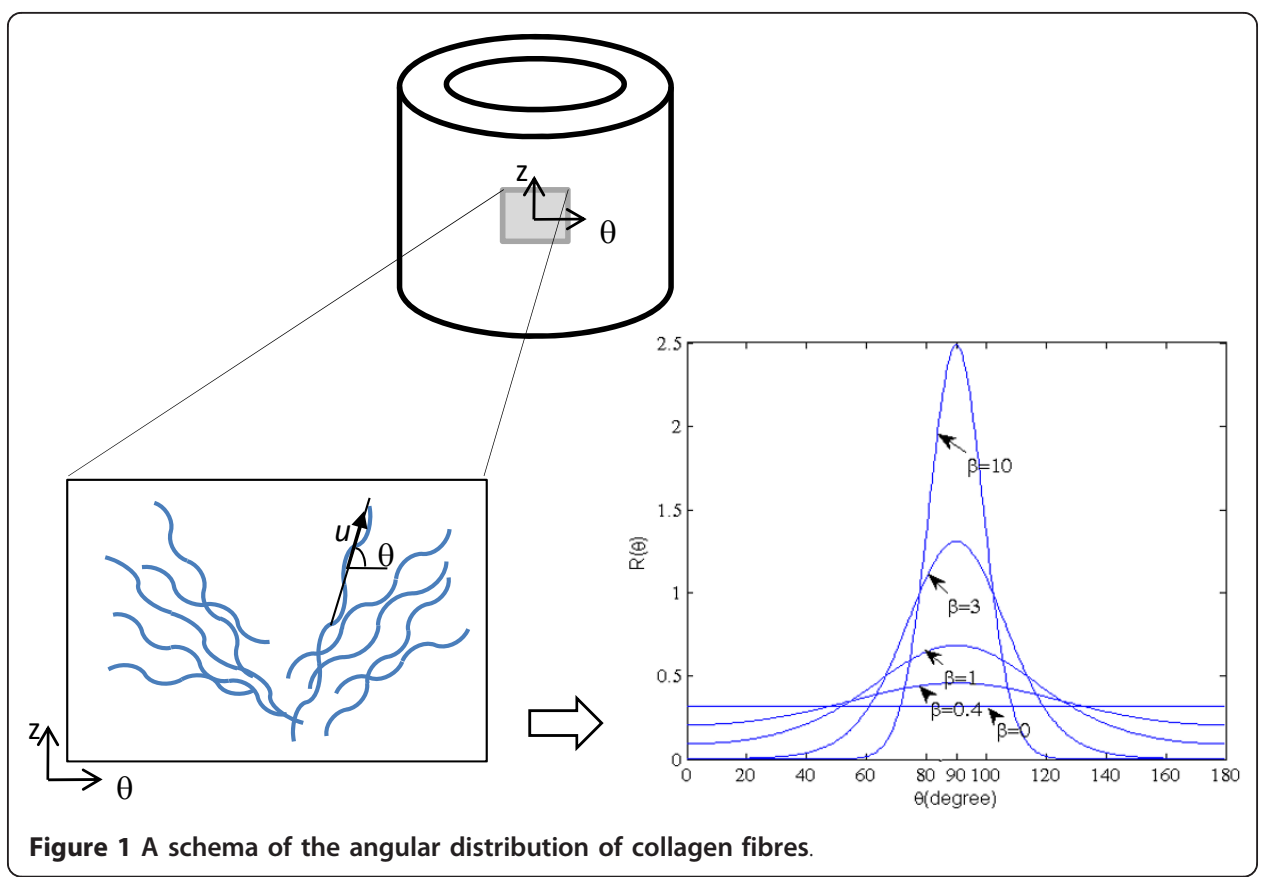

with the overall direction of the fibre [19] and makes an angle $\theta$ with respect to the circumferential direction. In each family of fibres, we assume that individual fibres follow a distribution around their mean orientation $\alpha$ in a statistical manner, with $R(\theta)$ being the angular distribution of a family of fibres. Therefore, $R(\theta) d \theta$ is the fraction of fibres oriented between $\theta$ and $\theta+d \theta$.

The collagen fibres are wavy. We assume that the load required to straighten fibres is negligible when compared to the load transmitted by the stretched fibres. Hence, collagen fibres transmit load only if stretched beyond the point where undulations disappear. This point is represented by $E^{*}$, the engagement strain in the direction of fibres, defined as:

$$
E^{*}=\frac{(\lambda *)^{2}-1}{2}
$$

where $\lambda^{*}$ is the stretch along the fibre at which the fibre straightens. We assume that for all fibres in the direction $\theta$, the engagement of the collagen fibres happens in some statistical manner [4,27]. A log-logistic probability distribution function [22,23] $\left(\rho_{\text {fibre }}\right)$ is chosen to account for the distribution of the engagement strain $E^{*}$ :

$$
\rho_{\text {fiber }}\left(E^{*}\right)= \begin{cases}0 & \text { for } E^{*}<0 \\ \frac{k}{b} \frac{\left(\frac{E^{*}}{b}\right)^{k-1}}{\left[1+\left(\frac{E^{*}}{b}\right)^{k}\right]^{2}} & \text { for } E^{*} \geq 0\end{cases}
$$

$b>0$ is a scaling parameter and $k>0$ defines the shape of the distribution. 
As stated above, we assume that fibres carry load only when stretched. For a bundle of straight fibres with the same overall direction, the strain energy function is defined by:

$$
\Psi_{\text {fiber }}\left(E^{f}\right)= \begin{cases}0 & \text { for } E^{f}<0 \\ \frac{1}{2} c_{\text {coll }}\left(E^{f}\right)^{2} & \text { for } E^{f} \geq 0\end{cases}
$$

where $c_{\text {coll }}$ is an elastic constant and $E^{f}$ is the local Green strain with respect to the fibre's straight configuration.

We assume that the distribution of the engagement pattern is independent of fibre orientation. This means that fibres oriented in any direction $\theta$, engage following the same probability density function $\rho_{\text {fibre }}$, as defined earlier. Let us consider a family of fibres oriented in the direction $\theta$ with respect to the circumferential direction. The green strain in the main direction of fibres is:

$$
E=E_{\theta} \cos ^{2} \theta+E_{z} \sin ^{2} \theta
$$

$E_{\theta}$ and $E_{z}$ are Green strains in the circumferential and longitudinal directions, respectively. The deformations have been calculated considering a circular cylindrical vessel whose zero-stress state is a circular sector [22,28].

At a certain Green strain $E$ along the fibres, the true strain in the fibre, with the straightening strain $E^{*}$, will be $\left(E-E^{*}\right) /\left(1+2 E^{*}\right)$ as explained in detail by Lokshin and Lanir [9]. Thus, the contribution of the fibres oriented between $\theta$ and $\theta+d \theta$ becomes:

$$
\Psi_{\text {coll }}^{\theta}(E)=\int_{0}^{E} \rho_{\text {fiber }}\left(E^{*}\right) \Psi_{\text {fiber }}\left(\frac{E-E^{*}}{1+2 E^{*}}\right) d E^{*}
$$

Consequently, the SEF of the ensemble of a family of fibres is defined as:

$$
\Psi_{\text {coll }}^{f}=\int_{0}^{\pi}\left(\int_{0}^{E} \rho_{\text {fiber }}\left(E^{*}\right) \Psi_{\text {fiber }}\left(\frac{E-E^{*}}{1+2 E^{*}}\right) d E^{*}\right) R(\theta) d \theta
$$

Thus the collagen SEF with half of the fibres having the angular distribution $R(\theta)$ with mean angle $\alpha$ and the other half having $R^{\prime}(\theta)$ with mean angle - $\alpha$ to the circumferential direction becomes:

$$
\Psi_{\text {coll }}=\frac{1}{2} \int_{0}^{\pi}\left(\int_{0}^{E} \rho_{\text {fiber }}\left(E^{*}\right) \Psi_{\text {fiber }}\left(\frac{E-E^{*}}{1+2 E^{*}}\right) d E^{*}\right) R(\theta) d \theta+\frac{1}{2} \int_{0}^{\pi}\left(\int_{0}^{E} \rho_{\text {fiber }}\left(E^{*}\right) \Psi_{\text {fiber }}\left(\frac{E-E^{*}}{1+2 E^{*}}\right) d E^{*}\right) R^{\prime}(\theta) d \theta
$$

\section{Form of angular distribution $R(\theta)$}

We assume that the orientation (main direction, as defined in Figure 1) of collagen fibres is distributed according to a planar $\pi$-periodic von-Mises distribution:

$$
R(\theta ; \alpha, \beta)=\frac{1}{\pi I_{0}(\beta)} e^{\beta \cos (2(\theta-\alpha))} \quad \text { for } 0 \leq \theta<\pi
$$

where $I_{0}$ denotes the modified Bessel function of the first kind and order 0, which can be defined by: 


$$
I_{0}(\beta)=\frac{1}{2 \pi} \int_{0}^{2 \pi} e^{\beta \cos \theta} d \theta
$$

This von-Mises distribution is a close approximation of a normal distribution wrapped on a circle. The parameter $\alpha$ is the mean direction and the parameter $\beta$ is known as the concentration parameter. The distribution is uni-modal and symmetrical about $\theta=\alpha$ [29].

The circular standard deviation $\sigma$ is defined as [29]:

$$
\sigma=\{-2 \log \rho\}^{1 / 2}
$$

where $\rho$ is the mean resultant length of the distribution. For the von-Mises distribution $\rho$ is equal to [29]:

$$
\rho=\frac{I_{1}(\beta)}{I_{0}(\beta)}
$$

where $I_{1}$ denotes the modified Bessel function of the first kind and order 1 :

$$
I_{1}(\beta)=\frac{1}{2 \pi} \int_{0}^{2 \pi} \cos \theta e^{\beta \cos \theta} d \theta
$$

The larger the value of $\beta$, the greater is the clustering around the mode. As an example, the distribution is illustrated in Figure 1 for mean value of $\alpha=90$ and $\beta$ values of $0,0.4,1,3$ and 10 . When $\beta=0$, the distribution $R(\theta ; \alpha, \beta)$ is uniform, meaning that there is no preferential angle for the ensemble of fibres. For larger values of $\beta$, the distribution becomes more concentrated around the mean angle $\alpha$.

Assuming that the two families of fibres are symmetric with respect to the longitudinal direction, we set $\alpha^{\prime}=-\alpha$ and we take the same concentration parameter $\beta$ for definition of $R^{\prime}(\theta)$, as used for $R(\theta)$.

\section{Parametric study of effects of mean collagen fibre angle and angle dispersion}

The parametric study was designed to elucidate the effect of concentration parameter $\beta$ and mean direction $\alpha$ of collagen fibres on the macro-mechanical behaviour of a vascular tube, which undergoes inflation-extension tests. The geometry of the reference (zero stress) state has been taken from our recent study on rabbit facial veins [26]. The opening angle was $115 \pm 12 \mathrm{deg}$ and average inner and outer arc lengths were $9.72 \pm$ $0.83 \mathrm{~mm}$ and $10.8 \pm 0.83 \mathrm{~mm}$, respectively. Elastic constant $c_{\text {colv }} c_{\text {elast }} c^{a}$ elast, and collagen engagement parameters $k$ and $b$ were taken from the best fit of the model with highly orientational fibres on the rabbit facial vein data [26]. Area fractions $f_{\text {elast }}$ and $f_{\text {coll }}$ were set to 0.10 and 0.48 as reported by the same study [26]. These parameters, used in the SEF, are listed in Table 1.

We studied the pressure-radius $\left(\mathrm{P}-\mathrm{r}_{\mathrm{o}}\right)$ and pressure-longitudinal force $\left(\mathrm{P}-\mathrm{F}_{\mathrm{z}}\right)$ response of the vessels using different sets of values for $\alpha$ and $\beta$. The mean orientation angle $\alpha$ $=34.2^{\circ}$ reflects the best fit for the set of experimental data on our previous study [26]. Having set $\alpha$ to $34.2^{\circ}$, we studied the effect of parameter $\beta$ on vessel response. 
Table 1 Values used for the parametric study

\begin{tabular}{ll}
\hline parameter & Fitted value \\
\hline$f_{\text {elast }}$ & 0.10 \\
$f_{\text {coll }}$ & 0.48 \\
$c_{\text {coll }}$ & $200 \mathrm{MPa}$ \\
$c_{\text {elast }}$ & $12392 \mathrm{~Pa}$ \\
$c^{a}{ }_{\text {elast }}$ & $7436 \mathrm{~Pa}$ \\
$k$ & 6.21 \\
$b$ & 3.26 \\
\hline
\end{tabular}

In addition to the "control value" i.e. $\alpha=34.2^{\circ}$, two other values of mean direction of fibres were chosen, a lower value $\alpha=14.2^{\circ}$ and a higher value $\alpha=54.2^{\circ}$. These values stand in the physiological range. For each of these mean direction angles, $\beta$ was set to $\beta=0,1,10$ and 1000. $\beta=0$ shows the uniform distribution of fibres (isotropic case) and $\beta=1000$ the highly concentrated distribution of fibres around the mean angle $\alpha$. For values of beta larger than 1000, we did not observe significant changes in the macro response of the tube (less than $1 \%$ difference). Therefore, for all practical purposes, we will refer to $\beta=1000$ as $\beta=\infty$.

\section{Fitting the model to experimental data}

To assess the effect of including a dispersion parameter on the quality of fits for P-r $\mathrm{r}_{\mathrm{o}}$ and $\mathrm{P}-\mathrm{F}_{\mathrm{z}}$ curves, the new model including dispersion is fit to the experimental data on rabbit facial veins. Parameters $c_{\text {elast }}^{i} c^{a}$ elast $, k, b, \alpha$ and $\beta$ were allowed to vary to be optimized in the curve fitting process. The elastic constant of collagen was chosen as $c_{\text {coll }}=200 \mathrm{MPa}$, a reasonable value taken from the literature and in accordance with Zulliger et al. [22]. Pressure-radius and pressure-longitudinal force curves were fitted to the experimental data by minimizing the following function using MATLAB R2007b (MATLAB, USA):

$$
\Phi=\frac{1}{2} \frac{1}{m} \sum_{i}^{m}\left(\frac{r_{i}^{\mathrm{mod}}-r_{i}^{\exp }}{\sigma_{i}^{r}}\right)^{2}+\frac{1}{2} \frac{1}{m} \sum_{i}^{m}\left(\frac{F_{i}^{\mathrm{mod}}-F_{i}^{\exp }}{\sigma_{i}^{F}}\right)^{2}
$$

$m$ is the number of experimental points measured at different pressures. Superscript mod denotes the values predicted by the mathematical model, whereas superscript exp shows those measured experimentally. The predictions of the outer radii and the longitudinal forces are obtained by integrating the equations of equilibrium and imposing the boundary conditions, as it is described in [26]. Index $i$ denotes the experimental points, i.e., the pairs of pressure and corresponding outer radius, $r$, and longitudinal force, F. $\sigma$ is the standard deviation of the experimental mean value of radius or force at a given pressure and longitudinal stretch ratio. It is used as a weighting factor, giving more weight to the points with the least variation. The $\Phi$ function has been used as a measure of the quality of the fit. Lower values of $\Phi$ are representing higher fit qualities.

The quality of the fit, based on the new model including fibre dispersion, was compared with the one with perfectly aligned fibres (no dispersion) [22]. This model is equivalent to $\beta=\infty$ referred to as the 'original model' in this article. For the model 
with no fibre angle dispersion, $c_{\text {coll }}$ was also taken equal to $200 \mathrm{MPa}[30,31]$ and parameters $c_{\text {elast }}^{i} c_{\text {elast }}^{a} k, b$ and $\alpha$ were allowed to vary freely to be optimized for best fit.

\section{Results}

\section{Parametric analysis}

Figure 2 shows the effect of dispersion parameter $\beta$ on $\mathrm{P}-\mathrm{r}_{\mathrm{o}}$ and $\mathrm{P}-\mathrm{F}_{\mathrm{z}}$ curves, with the elastic constants being the same as the ones obtained by minimizing $\Phi$ using the original model (without dispersion). Columns a, b and c represent the model predictions for $\alpha$ equal to 14.2, 34.2 and 54.2 degrees respectively. The solid curve in Figure 2 plots the response of the tube for $\beta=0$ (the isotropic case in which orientation of collagen fibers is distributed uniformly). Our results showed that for values of $\beta$ higher than 1000 (circular standard deviation $\sigma$ less than $1.8^{\circ}$ ) the radius and longitudinalforce values changed only slightly (less than $1 \%$ ). Therefore, the value $\beta=1000$ has been used for the case of highly oriented fibres (dashed line - -) and referred to as $\beta=\infty$ in the figures. The effect of concentration parameter $\beta$ on $\mathrm{P}-\mathrm{r}_{\mathrm{o}}$ and $\mathrm{P}-\mathrm{F}_{\mathrm{z}}$ curves seemed to depend on the mean orientation. As seen from Figure 2, for $\alpha=14.2$, the curves for $\beta=10$ and $\beta=\infty$ almost overlap. For instance, at $\mathrm{P}=2 \mathrm{KPa}(15 \mathrm{mmHg}), \mathrm{r}_{\mathrm{o}}$ is 1.781 for $\beta=10$ compared to $1.780 \mathrm{~mm}$ for $\beta=\infty$ showing a difference of less than $0.1 \%$. The variations in $\mathrm{F}_{\mathrm{z}}$ are also less than $1 \%$. However, for $\alpha=54.2$, the effect of concentration parameter $\beta$ becomes much more important. For the same pressure value ( $2 \mathrm{KPa}$ ), when $\alpha$ is fixed to $54.2^{\circ}, \mathrm{r}_{\mathrm{o}}$ is equal to $2.19 \mathrm{~mm}$ for $\beta=10$ compared to $2.37 \mathrm{~mm}$ for $\beta=\infty$ showing a decrease of around $8 \%$ in radius. As for the longitudinal force, $\mathrm{F}_{\mathrm{z}}$ decreases from $9.17 \mathrm{mN}$ for $\beta=\infty$ to $2.88 \mathrm{mN}$ for $\beta=10$, a difference of more than $69 \%$.

$\beta=0$ is the equivalent of an isotropic distribution of the orientation of collagen fibres. Therefore, for $\beta=0$ the different mean fibre orientations (indicated by different angles $\alpha$ ) lose their meaning and the response of the tube is isotropic (solid curves in

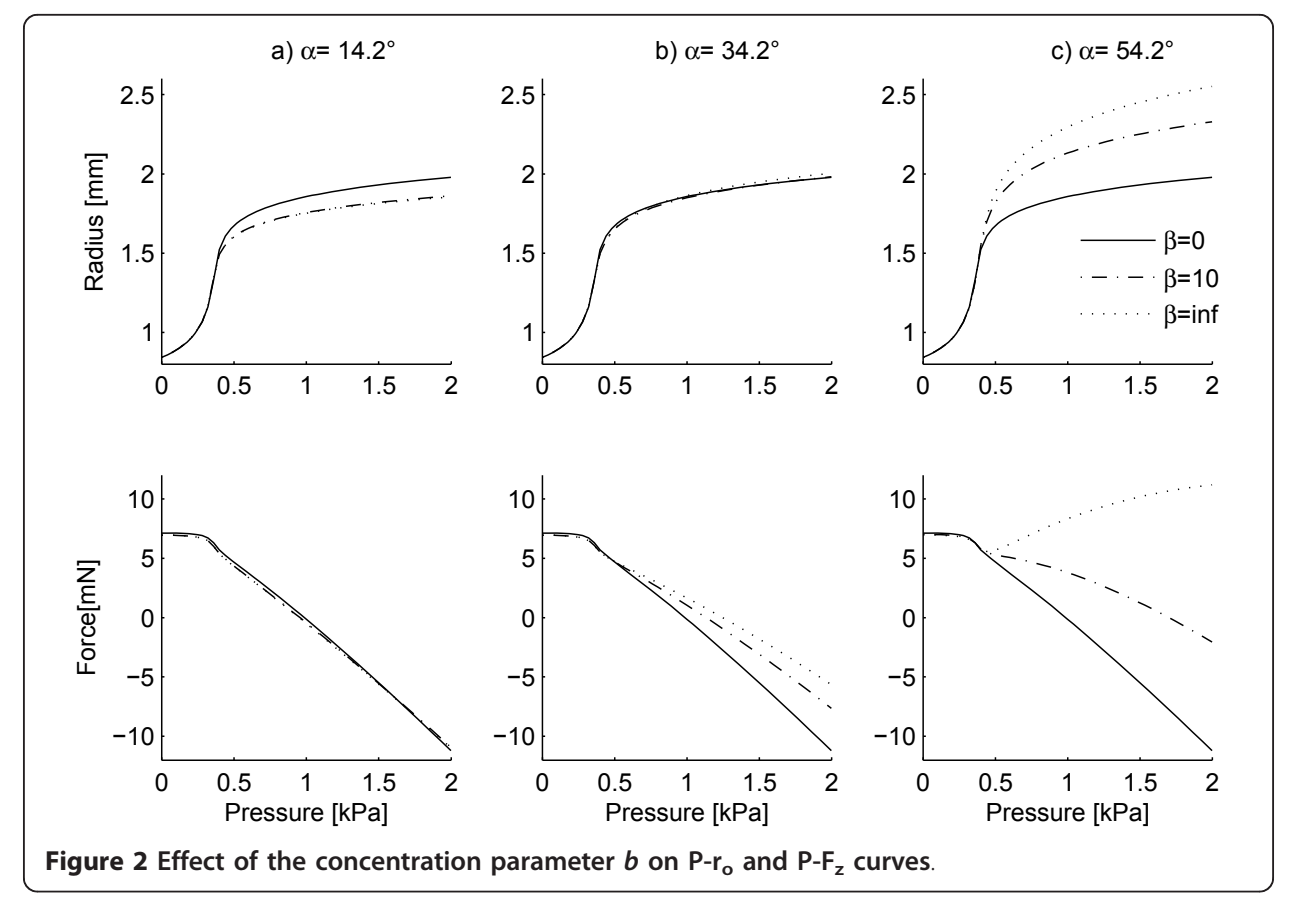


Figure 2). On the contrary, for values of $\beta=1,10$ and $1000(\infty)$ plotted in Figure 3 the macro-mechanical response of the tissue depends on the mean orientation of the fibres. Based on Figure 3, the response of the material with highly oriented fibres $(\beta=\infty)$ depends strongly on the mean orientation angle $\alpha$. For example, at $\mathrm{P}=2 \mathrm{kPa}$ $\left(15 \mathrm{mmHg}\right.$ ) and $\alpha=34.2^{\circ}$, the radius and longitudinal pressure are $\mathrm{r}_{\mathrm{o}}=1.90 \mathrm{~mm}$ and $\mathrm{F}_{\mathrm{z}}=0.323 \mathrm{mN}$ respectively. Increasing the mean alignment to $\alpha=54.2^{\circ}$ gives values of $\mathrm{r}_{\mathrm{o}}=2.37 \mathrm{~mm}$ and $\mathrm{F}_{\mathrm{z}}=9.17 \mathrm{mN}$ showing $25 \%$ increase in radius and $2740 \%$ increase in longitudinal force.

\section{Fitting the model to experimental data}

Figure 4 shows the best fit for the experimental data on the medial layer of rabbit facial veins using the original model (with all fibres in a family of fibres aligned in one direction) as well as the modified model, which includes the dispersion of collagen fibres. The values used to fit the data are shown in Table 2. As seen from Figure 4 and Table 2, for this set of data, the quality of fits is almost the same $(\Phi=0.188$ for the original model compared to $\Phi=0.181$ for the modified model), hence, for this particular vessel including dispersion in the collagen fibres does not seem to improve the quality of the fit.

\section{Discussion}

In this study, a structural constitutive model for the macro-mechanical behaviour of vascular tissue is presented based on the framework developed by Lanir et al. [19,20]. The new model incorporates both waviness and orientational distribution of collagen fibres. The waviness of fibres is modelled by a log-logistic distribution [22,23]. To account for the angular distribution of fibres, we suggested a planar $\pi$-periodic vonMises distribution with mean orientation $\alpha$ and concentration parameter $\beta$. We studied the effect of these parameters $(\alpha$ and $\beta$ ) on the macroscopic vessel response to

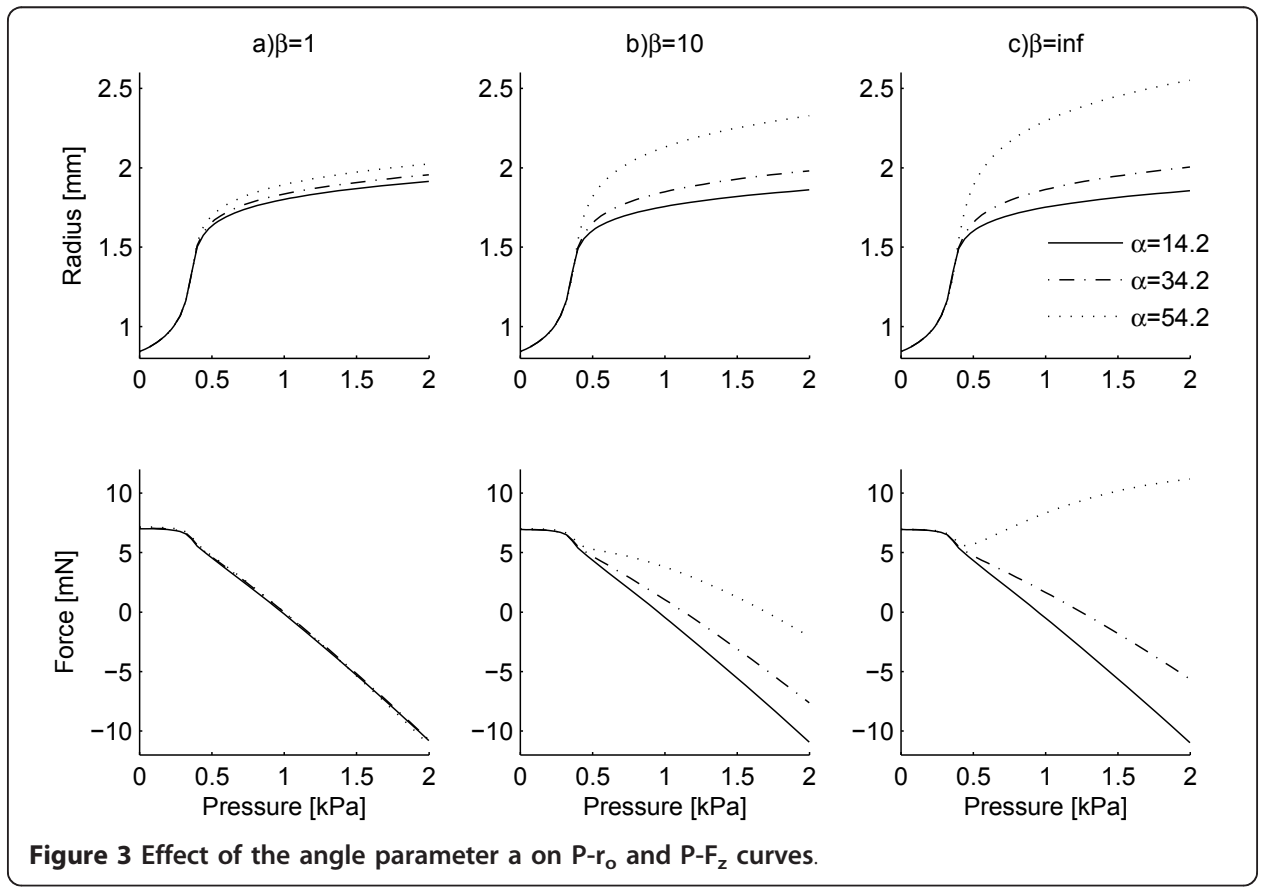



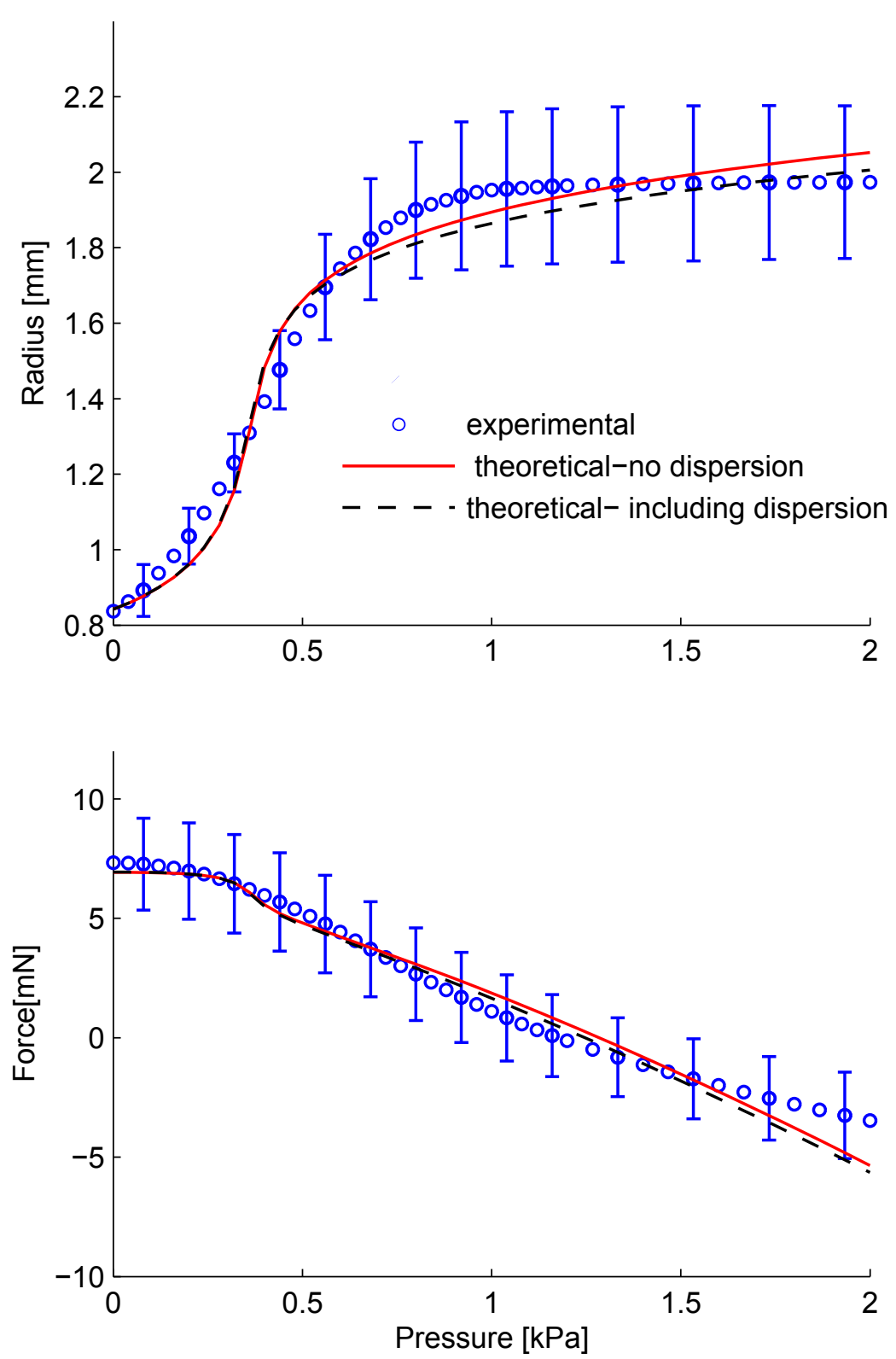

Figure 4 Best fits to experimental data from inflation-extension tests. Best fit to experimental data from the original model with no dispersion $\left(\_\right.$) and the modified model including dispersion (- - - )

inflation-extension tests, typically expressed as $\mathrm{P}-\mathrm{r}_{\mathrm{o}}$ and $\mathrm{P}-\mathrm{F}_{\mathrm{z}}$ curves. Finally, the new model was applied to fit experimental data of inflation-extension tests of rabbit facial veins in order to assess the usefulness or necessity of including the fibre angle dispersion in the model.

\section{The choice of strain energy function for elastin}

In the present work the SEF for elastin used is a combination of Neo-Hookean SEF with a SEF describing a family of fibres in the longitudinal direction. In our previous work [26] the anisotropic SEF for elastin led to good representation of both the pressure-diameter and pressure-force curves. The anisotropic properties of elastin have 
Table 2 Parameters used in minimizing $\Phi$

\begin{tabular}{lll}
\hline Strain energy function & $\begin{array}{l}\text { Original model (without } \\
\text { dispersion) }\end{array}$ & $\begin{array}{l}\text { modified model (with } \\
\text { dispersion) }\end{array}$ \\
\hline Fitted parameters & $c_{\text {elast }}^{j}=12392 \mathrm{~Pa}$ & $c_{\text {elast }}^{i}=12468 \mathrm{~Pa}$ \\
& $c^{a}$ elast $=7436 \mathrm{~Pa}$ & $c^{a}$ elast $=7344 \mathrm{~Pa}$ \\
$k=6.21$ & $k=5.87$ \\
& $b=3.26$ & $b=3.53$ \\
& $\alpha=34.2^{\circ}$ & $\alpha=36.2^{\circ}$ \\
& & $\beta=217.73$ \\
\hline Experimentally defined & $f_{\text {elast }}=0.10$ & $f_{\text {elast }}=0.10$ \\
parameters & $f_{\text {coll }}=0.48$ & $f_{\text {coll }}=0.48$ \\
\hline Imposed on model & $c_{\text {coll }}=200 \mathrm{MPa}$ & $c_{\text {coll }}=200 \mathrm{MPa}$ \\
\hline$\Phi$ & 0.188 & 0.181 \\
\hline
\end{tabular}

been confirmed for several types of tissues. In [32] histological data from distal carotid and femoral arteries showed longitudinal arrangement of elastin in the adventitia of these tissues. The anisotropic behaviour of elastin is also supported by the experimental works $[33,34]$. In the former, they performed both uniaxial tests of strips and inflation tests, and in the latter they performed biaxial tensile tests. Both experimental works showed that elastin was stiffer in circumferential than in longitudinal direction. From these experimental results, the choice of an anisotropic SEF for elastin appears reasonable.

\section{The choice of strain energy function for collagen}

Various structural energy functions have been developed previously to account for collagen microstructure in cardiovascular tissue. In this study, we modelled both waviness and orientational distribution of collagen fibres. Collagen fibres appear to be coiled and wavy in their unloaded state $[14,15]$ and form two helically arranged families of fibres. The individual collagen fibres, in each family, show a deviation from their mean orientations [16,17]. Similar to our approach, some of previous studies also followed the work by Lanir et al. $[19,20]$. Billiar and Sacks proposed a model for aortic valve cusps and introduced the orientational distribution of collagen fibres to their model by means of a Gaussian Distribution [24]. Sacks extended the previous work and included further the pattern of recruitment of fibres in the model using a gamma distribution [10]. As for the orientational distribution of collagen fibres, differently from their earlier work, Sacks proposed a beta distribution and the modified model was applied on biaxial tests of Bovine pericardium. Other studies followed a different approach involving the use of invariants and introducing concepts of waviness, as the studies of Zulliger et al. [23] and Cacho et al. [35], or orientational distribution, as in the works of Gasser et al. and Driessen et al. [11,25]. In [36], Desch et al. developed a 2D constitutive model based on their observation that the ratio of the circumferential to the longitudinal first Piola-Kirchoff stress was a function of the circumferential stretch ratio. They proposed a decomposition of the axial stretch ratio in an "elastic" and an "inelastic" part. Alastrue et al. [37] proposed a phenomenological SEF which consists of an isotropic part (Neo-Hookean SEF) and an anisotropic part with two anisotropy directions (longitudinal and circumferential). They implemented their model on experimental data obtained by uniaxial tension tests on vena cava strips in longitudinal and 
circumferential directions. In [38-40] a four-family fibre model was used, in which the orientations of the fibres were the circumferential, the longitudinal and the two diagonals. In the experimental work [41] on thoracic aortic segments of rabbits two distinct sets of collagen were found; the first was in the form of bundles with predominantly circumferential direction, and the second in the form of a pericellular matrix with interlaced fibrils. The collagen bundles were found to have a circumferential orientation, whereas the interlaced fibrils of the pericellular matrix were found straightened along both the radial and longitudinal directions.

In the present study, we used a log-logistic distribution to account for waviness and the gradual engagement of the fibres, similar to the study of Zulliger et al. [23]. The log-logistic probability distribution used in the present study has a lower bound that satisfies the hypothesis that in the zero-stress state no fibres are strained. The choice of the log-logistic probability distribution is based on a phenomenological approach and the precise distribution of the fibre engagement remains to be determined by other appropriate experimental studies. The present formulation to model the waviness is, however, slightly different from the one suggested by Zulliger et al. Zulliger and colleagues suggested that the waviness could be modelled by a convolution resulting in a $\Psi_{\text {coll }}^{\theta}$ as:

$$
\Psi_{\text {coll }}^{\theta}(E)=\rho_{\text {fiber }} * \Psi_{\text {fiber }}=\int_{0}^{E} \rho_{\text {fiber }}\left(E^{*}\right) \Psi_{\text {fiber }}\left(E-E^{*}\right) d E^{*}
$$

On the contrary, the present study models the waviness as:

$$
\Psi_{\text {coll }}^{\theta}(E)=\int_{0}^{E} \rho_{\text {fiber }}\left(E^{*}\right) \Psi_{\text {fiber }}\left(\frac{E-E^{*}}{1+2 E^{*}}\right) d E^{*}
$$

The difference resides in the term $\left(E-E^{*}\right) /\left(1+2 E^{*}\right)$ which expresses the true strain in the group of fibres that are engaged at the Green strain $E^{*}$. For more details, refer to the work by Lokshin and Lanir [9].

As for the orientational distribution, we made the assumption that fibres are mainly in the circumferential-longitudinal plane and we have chosen a planar von-Mises probability density function (PDF) to express the angular distribution of fibres. The assumption that collagen fibres lay preferentially in the circumferential-longitudinal plane seems reasonable based on values reported in the literature for the radial component of collagen fibres. Finlay et al. reported radial angle of around $5^{\circ}$ in the adventitia and $8^{\circ}$ in the media for collagen in human brain arteries fixed at $30 \mathrm{mmHg}$ [17]. In addition, Canham et al. measured this value to be $2^{\circ}$ in the media and $1^{\circ}$ in the adventitia of human saphenous veins, fixed at $110 \mathrm{mmHg}$ [42]. As for the choice of orientational distribution, Gasser et al. have used similarly the von-Mises distribution for orientational distribution of collagen fibres with the difference that they assumed a spatial distribution [25] while Billiar and Sacks chose a Gaussian distribution [24]. The choice of Gaussian over von-Mises distribution does not seem to affect significantly the model since any von-Mises distribution can be approximated by a (wrapped) Gaussian distribution [29]. Certainly, more experimental work on orientational distribution as well as the waviness of collagen fibres in various tissues is needed to quantify fibres' structure and clarify the type of the distributions to be used in structural models. 
In literature there are few studies that combine biomechanical and histometrical data in order to support the association between the mechanical properties of arterial/vein tissue and histology. The findings of the experimental work of Wicker et al. [40] suggested medial collagen highly aligned about a circumferential direction, adventitial collagen widely distributed about a mean axial direction, as well as a helical orientation for the adventitial layer. Based on these experimental findings they used a four-fibre family model in order to describe the contribution of collagen. For the contribution of the elastin-dominated amorphous extracellular matrix they used a Neo-Hookean SEF. Another study that combines histological and biomechanical data is [32]. For the tissue studied there, Sokolis' histological data indicated orthotropic symmetry for unstressed elastin with some types of arteries exhibiting a prominent elastin component in their adventitia with longitudinal arrangement. The medial collagen was observed in the form of lengthy crimped fibre-bundles with circumferential orientation and a fibril network encasing circumferentially directed cellular elements. In addition to these findings the waviness of elastin and collagen fibres was different in each direction, and type of artery dependent. In order to describe the mechanical response of the passive state of the tissue he used a quadratic and exponential SEF. Although he used a phenomenological SEF, the choice of the quadratic term was in accordance with the histological finding of orthotropic symmetry of unstressed elastin, and he provided a correlation of the SEF parameters with histological data (composition, orientation, and waviness of elastin and collagen fibres).

In this study, the vascular wall is for simplicity modelled as a one-layer material. However, the blood vessel is composed of different layers and the composition and arrangement of intramural wall components differ from one layer to another. For instance, in the media, collagen fibres are circumferentially and coherently aligned, whereas in the adventitia, the pitch of the helically arranged fibres and the dispersity of the fibre distribution increases $[17,42]$. Obviously, the present model assumes homogenized properties throughout the vascular wall and therefore would be more accurate when only applied to single layer structures. In future studies, a two-layer structure model which integrates both waviness and dispersion of collagen fibres should be developed. Furthermore, since collagen type in the media (primarily of Type I, III and $\mathrm{V}$ ) is different from the adventitia (primarily of type I) [2], future studies should also include a different collagen elastic constant for each layer.

\section{Effect of orientational distribution parameters $\alpha$ and $\beta$ on gross material response}

The results of our parametric study showed that the effect of concentration parameter $\beta$ on $\mathrm{P}-\mathrm{r}_{\mathrm{o}}$ and $\mathrm{P}-\mathrm{F}_{\mathrm{z}}$ curves depends on the mean orientation of fibres $\alpha$ (Figure 2). The model is less sensitive to adding orientational dispersion when fibres are located close to the circumferential direction (lower mean orientation of fibres $\alpha$ ) than when fibres are located further from the circumferential direction (higher mean orientation of fibres). For instance, when $\alpha$ was fixed at $14.2^{\circ}$ and a circular standard deviation $\sigma$ of $18.6^{\circ}$ was applied $(\beta=10), \mathrm{r}_{\mathrm{o}}$ and $\mathrm{F}_{\mathrm{z}}$ did not change more than $1 \%$ compared to the case without dispersion $\left(\sigma=0\right.$ i.e. $\beta=\infty$ ). However, at $\alpha=54.2^{\circ}$, the $\mathrm{P}-\mathrm{r}_{\mathrm{o}}$ and $\mathrm{P}-\mathrm{F}_{\mathrm{z}}$ curves deviated $8 \%$ in $r_{\mathrm{o}}$ and $69 \%$ in $\mathrm{F}_{\mathrm{z}}$ compared to the case without dispersion.

The higher values of mean orientation of fibres $\alpha$ are generally associated with the adventitia [17] while lower mean orientations are associated with the media [16]. 
Therefore, considering our results, one could conclude that in the presence of the adventitia, the model should include the information about the dispersion of collagen fibres and the effect of dispersion could not be neglected. While when the adventitia is removed (only the media is present), one could use a simpler model that does not include angular dispersion of collagen.

Our results showed that the material response is highly sensitive to the mean orientation of fibres $\alpha$ when fibres are highly oriented (large values of $\beta$ i.e. low values of $\sigma$ ) which is the case in the media. On the other hand, when fibres are dispersed, which is the case in the adventitia, the material response is less sensitive to the mean orientation of fibres. Gasser et al. have observed the same effect in their study [25].

\section{Fitting the model to experimental data}

In the present study, the original model of Zulliger et al. (no dispersion, $\sigma=0$ ) as well as our new model (including dispersion) were fitted to the set of experimental data from a previous study on rabbit facial veins in which the adventitia was removed mechanically [26]. When our model including dispersion was fitted to the data, the best fit was achieved with the concentration parameter $\beta=144\left(\sigma=4.8^{\circ}\right)$. As the adventitia was removed from the tissue, this value would represent the dispersion of collagen fibres in the media. Canham et al. have previously reported the circular standard deviation $\sigma$ of fibres in the media to be $5.2^{\circ}$ in brain arteries and $5.6^{\circ}$ in coronary arteries [18]. The results value from our model is therefore similar with those of Canham et al. Because different type of tissue has been used there, the result of the present work provides guidance for future experiments performed on rabbit facial veins. In these experiments one needs to examine the main orientation of collagen fibres, e.g. parameter $\alpha$ of our model, measure the angular distribution of the fibres, and fit a suitable distribution function to these measurements. Let a planar $\pi$-periodic von-Mises distribution be the suitable one, parameter $\beta$ of our model will be determined.

Including dispersion of collagen fibres resulted in almost the same quality of the simultaneous fit of the $\mathrm{P}-\mathrm{r}_{\mathrm{o}}$ and $\mathrm{P}-\mathrm{F}_{\mathrm{z}}$ curves as the original model of Zulliger et al. ( $\Phi$ $=0.188$ for the original model compared to $\Phi=0.181$ for the modified model) which shows that in case the adventitia is removed, the simpler model of Zulliger et al. could be used. However, the new model (including dispersion) resulted in a slightly higher (6\%) mean orientation of fibres $\alpha$ as well as an earlier and less abrupt engagement pattern of collagen fibres. The log-logistic probability distribution functions, $\rho_{\text {fibre }}$, for both models, with and without dispersion, have been plotted in Figure 5 using the values of $k$ and $b$ from Table 2 . The probability distribution function $\rho_{\text {fibre }}$ becomes concentrated for smaller values of $b$ and gets more spread out for larger ones. Based on Table 2, the value of $b$ is smaller in the original model (without dispersion) than the modified one (including dispersion). Therefore, the original model without dispersion shows a more concentrated distribution and thus a more abrupt engagement.

In addition, the mode (peak value) of the log-logistic distribution is situated at $b\left(\frac{k-1}{k+1}\right)^{1 / k}$. Therefore, the peak happens earlier (at $E=3.09$ ) in the original model without dispersion than the modified one including dispersion (at $\mathrm{E}=3.45$ ), resulting in an earlier engagement of collagen fibres in the Zulliger et al. model than the modified one. The results suggest that including the orientational dispersion of collagen 


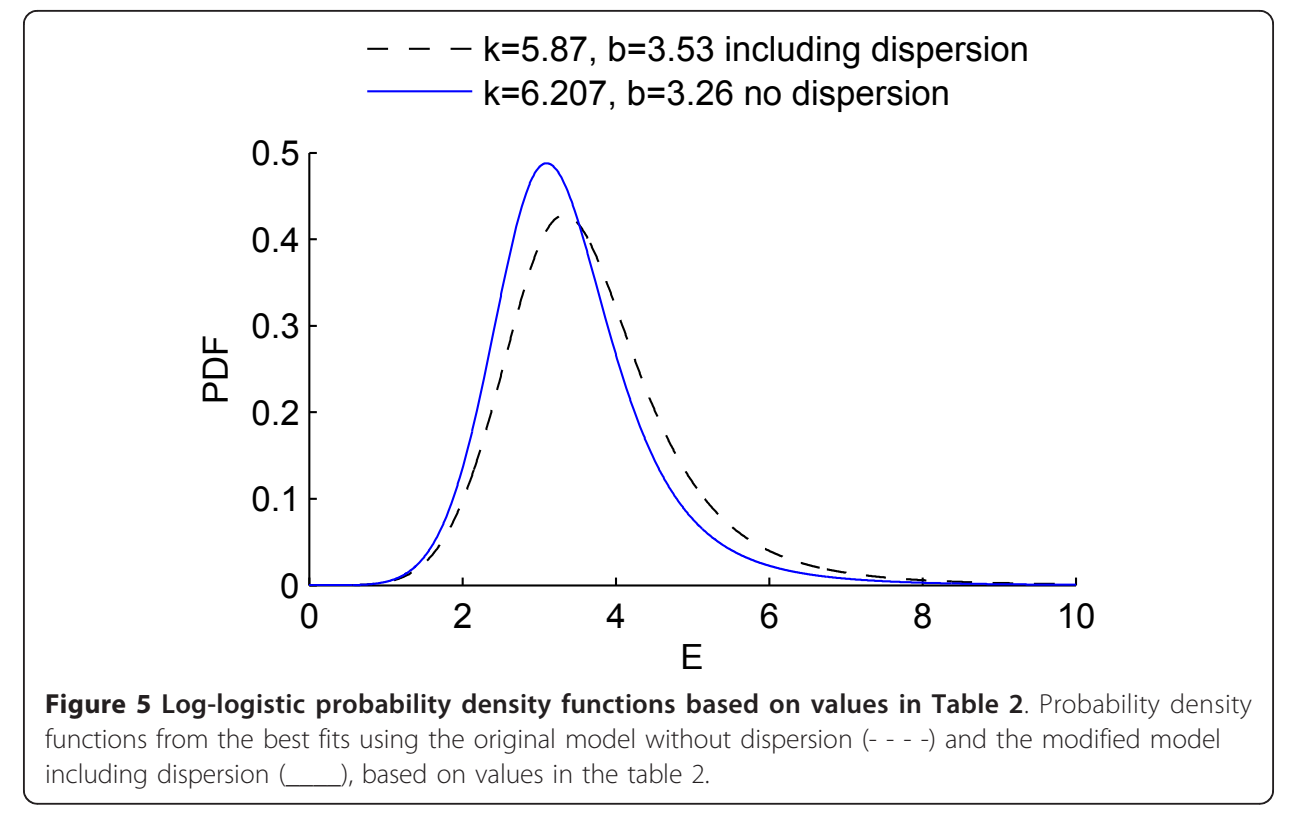

fibres changes the parameters of the collagen engagement pattern. This should be particularly considered when fit parameters are used to study changes in the engagement pattern $[43,44]$ and/or orientational distribution of collagen fibres [45]. As an example, assume that the orientational distribution of collagen fibres has been changed as a result of a mechanically induced remodelling. A model which does not include the dispersion of fibres could result in an 'unrealistic' change in parameters related to engagement pattern of collagen fibres.

\section{Conclusions}

In summary, we developed a strain energy function for vascular tissue considering both waviness and orientational distribution of collagen fibres and studied effects of parameters related to the orientational distribution of collagen fibres. We conclude that in the presence of the adventitia, the model should include the dispersion of collagen, while when the adventitia is removed (only the media is present), one could use a simpler model that does not include angular dispersion of collagen. In addition, it is very important to consider the dispersion of fibres when structural changes of collagen fibres, such as the collagen engagement pattern or orientation of fibres, are studied. Despite its limitations, the model offers possibilities to better understand the relation between structure and function in the vascular wall and to further study mechanically induced collagen remodelling in vascular tissue in health and disease. This can be achieved by relating fibre turnover, reorientation and waviness reorganization to the mechanical loading conditions. 
designed the study, participated in interpretation of data and revised the manuscript critically. All authors read and approved the final manuscript.

\section{Competing interests}

The authors declare that they have no competing interests.

Received: 16 August 2010 Accepted: 4 March 2011 Published: 4 March 2011

\section{References}

1. Fung YC: Biomechanics: Mechanical Properties of living tissues Springer-Verlag; 1981.

2. Humphrey JD: Cardiovascular Solid Mechanics: cells, tissues and organs New York: Springer-Verlag New York; 2002.

3. Holzapfel GA, Gasser TC, Stadler M: A structural model for the viscoelastic behavior of arterial walls: Continuum formulation and finite element analysis. European Journal of Mechanics, A/Solids 2002, 21:441-463.

4. Wuyts FL, Vanhuyse VJ, Langewouters GJ, Decraemer WF, Raman ER, Buyle S: Elastic properties of human aortas in relation to age and atherosclerosis: A structural model. Phys Med Biol 1995, 40:1577-1597.

5. Zulliger MA, Stergiopulos N: Structural strain energy function applied to ageing and hypertension. Arch Physiol Biochem 2004, 112:67.

6. Alastrue V, Martinez MA, Doblare M, Menzel A: Anisotropic micro-sphere-based finite elasticity applied to blood vessel modelling. Journal of the Mechanics and Physics of Solids 2009, 57:178-203.

7. Machyshyn IM, Bovendeerd PH, van de Ven AA, Rongen PM, van de Vosse FN: A model for arterial adaptation combining microstructural collagen remodeling and 3D tissue growth. Biomech Model Mechanobiol 2010, 9:671-687.

8. van den Broek $C$, van der Horst A, Rutten M, van de Vosse F: A generic constitutive model for the passive porcine coronary artery. Biomechanics and Modeling in Mechanobiology .

9. Lokshin O, Lanir Y: Micro and macro rheology of planar tissues. Biomaterials 2009, 30:3118-3127.

10. Sacks MS: Incorporation of experimentally derived fiber orientation into a structural constitutive model for planar collagenous tissues. J Biomech Eng 2003, 125:280-287.

11. Driessen NJB, Bouten CVC, Baaijens FPT: A structural constitutive model for collagenous cardiovascular tissues incorporating the angular fiber distribution. J Biomech Eng 2005, 127:494-503.

12. Hurschler C, Loitz-Ramage B, Vanderby R Jr: A structurally based stress-stretch relationship for tendon and ligament. J Biomech Eng 1997, 119:392-399.

13. Federico S, Gasser TC: Nonlinear elasticity of biological tissues with statistical fibre orientation. J R Soc Interface 2010 7:955-966.

14. Clark JM, Glagov S: Transmural Organization Of The Arterial Media - The Lamellar Unit Revisited. Arteriosclerosis 1985, 5:19-34.

15. Dingemans KP, Teeling P, Lagendijk JH, Becker AE: Extracellular matrix of the human aortic media: an ultrastructural histochemical and immunohistochemical study of the adult aortic media. Anat Rec 2000, 258:1-14.

16. Canham PB, Finlay HM, Dixon JG, Boughner DR, Chen A: Measurements from light and polarised light microscopy of human coronary arteries fixed at distending pressure. Cardiovasc Res 1989, 23:973-982.

17. Finlay HM, McCyllough L, Canham PB: Three-dimensional collagen organization of human brain arteries at different transmural pressures. J Vasc Res 1995, 32:301-312.

18. Canham PB, Talman EA, Finlay HM, Dixon JG: Medial collagen organization in human arteries of the heart and brain by polarized light microscopy. Connect Tissue Res 1991, 26:121-134.

19. Lanir Y: Constitutive equations for fibrous connective tissues. J Biomech 1983, 16:1-12

20. Lanir Y: A structural theory for the homogeneous biaxial stress-strain relationships in flat collagenous tissues. J Biomech 1979, 12:423-436.

21. Lanir $Y$, Lichtenstein $O$, Imanuel $O$ : Optimal design of biaxial tests for structural material characterization of flat tissues. J Biomech Eng 1996, 118:41-47.

22. Zulliger MA, Fridez $P$, Stergiopulos N, Hayashi $K$ : A strain energy function for arteries accounting for wall composition and structure. J Biomech 2004, 37:989-1000.

23. Zulliger MA, Rachev A, Stergiopulos N: A constitutive formulation of arterial mechanics including vascular smooth muscle tone. Am J Physiol Heart Circ Physiol 2004, 287:H1335-1343.

24. Billiar KL, Sacks MS: Biaxial mechanical properties of the native and glutaraldehyde-treated aortic valve cusp: Part II-A structural constitutive model. J Biomech Eng 2000, 122:327-335.

25. Gasser TC, Ogden RW, Holzapfel GA: Hyperelastic modelling of arterial layers with distributed collagen fibre orientations. Journal of the Royal Society Interface 2006, 3:15-35.

26. Rezakhaniha R, Stergiopulos N: A structural model of the venous wall considering elastin anisotropy. J Biomech Eng 2008, 130.

27. Decraemer WF, Maes MA, Vanhuyse VJ: An elastic stress-strain relation for soft biological tissues based on a structural model. J Biomech 1980, 13:463-468.

28. Fung YC: Biomechanics: motion, flow, stress and growth New York: Springer-Verlag New York Inc; 1990.

29. Mardia KV, Jupp PE: Directional statistics Chichester, John Wiley; 2000

30. Burton AC: Relation of structure to function of the tissues of the wall of blood vessels. Physiol Rev 1954, 34:619-642.

31. Milnor WR: Hemodynamics Baltimore: William \& Wilkins; 1989.

32. Sokolis DP: A passive strain-energy function for elastic and muscular arteries: correlation of material parameters with histological data. Med Biol Eng Comput 48:507-518.

33. Lillie MA, Shadwick RE, Gosline JM: Mechanical anisotropy of inflated elastic tissue from the pig aorta. J Biomech 43:2070-2078.

34. Zou Y, Zhang Y: An experimental and theoretical study on the anisotropy of elastin network. Ann Biomed Eng 2009, 37:1572-1583.

35. Cacho F, Elbischger PJ, Rodriguez JF, Doblare M, Holzapfel GA: A constitutive model for fibrous tissues considering collagen fiber crimp. International Journal of Non-Linear Mechanics 2007, 42:391-402. 
36. Desch GW, Weizsacker HW: A model for passive elastic properties of rat vena cava. J Biomech 2007, 40:3130-3145.

37. Alastrue V, Pena E, Martinez MA, Doblare M: Experimental study and constitutive modelling of the passive mechanical properties of the ovine infrarenal vena cava tissue. J Biomech 2008, 41:3038-3045.

38. Gleason RL, Dye WW, Wilson E, Humphrey JD: Quantification of the mechanical behavior of carotid arteries from wild-type, dystrophin-deficient, and sarcoglycan-delta knockout mice. J Biomech 2008, 41:3213-3218.

39. Wan W, Yanagisawa H, Gleason RL Jr: Biomechanical and microstructural properties of common carotid arteries from fibulin-5 null mice. Ann Biomed Eng 38:3605-3617.

40. Wicker BK, Hutchens HP, Wu Q, Yeh AT, Humphrey JD: Normal basilar artery structure and biaxial mechanical behaviour. Comput Methods Biomech Biomed Engin 2008, 11:539-551.

41. Sokolis DP, Kefaloyannis EM, Kouloukoussa M, Marinos E, Boudoulas H, Karayannacos PE: A structural basis for the aortic stress-strain relation in uniaxial tension. J Biomech 2006, 39:1651-1662.

42. Canham PB, Finlay HM, Boughner DR: Contrasting structure of the saphenous vein and internal mammary artery used as coronary bypass vessels. Cardiovasc Res 1997, 34:557-567.

43. Fonck E, Prod'hom G, Roy S, Augsburger L, Rufenacht D, Stergiopulos N: Effect Of Elastin Degredation on carotid wall mechanics as assessed by a constituent-based biomechanical model. American Journal of Physiology - Heart and Circulatory Physiology 2007, 292:H2754-H2763.

44. Roy S, Silacci P, Stergiopulos N: Biomechanical proprieties of decellularized porcine common carotid arteries. American Journal of Physiology - Heart and Circulatory Physiology 2005, 289:H1567-H1576.

45. Driessen NJB, Cox MAJ, Bouten CVC, Baaijens FPT: Remodelling of the angular collagen fiber distribution in cardiovascular tissues. Biomechanics and Modeling in Mechanobiology 2007, 7:93-103.

doi:10.1186/1475-925X-10-18

Cite this article as: Agianniotis et al:: A structural constitutive model considering angular dispersion and waviness

of collagen fibres of rabbit facial veins. BioMedical Engineering OnLine 2011 10:18.

\section{Submit your next manuscript to BioMed Central and take full advantage of:}

- Convenient online submission

- Thorough peer review

- No space constraints or color figure charges

- Immediate publication on acceptance

- Inclusion in PubMed, CAS, Scopus and Google Scholar

- Research which is freely available for redistribution 